\title{
Obtención de polvo de aluminio por atomización $^{(\cdot)}$
}

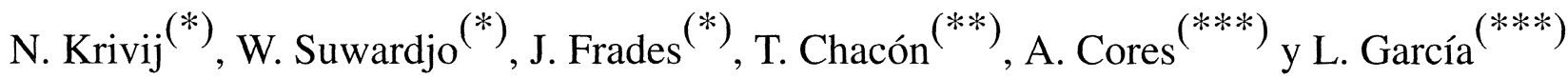 \\ Resumen En una planta piloto de pulvimetalurgia se obtiene polvo de aluminio destinado a la fabricación de \\ piezas sinterizadas. Se determina la distribución granulométrica y se realizan la caracterización \\ tecnológica y el estudio morfométrico del polvo de aluminio. Se estudia la sinterabilidad del polvo \\ de aluminio mediante la sinterización isotérmica de las muestras prensadas a diferentes temperaturas \\ y tiempos de retención.
}

Palabras clave: Polvo de aluminio. Atomización. Pulvimetalurgia.

\section{Obtaining of aluminium powder by atomization}

\begin{abstract}
In a powder metallurgy pilot plant aluminium powder for the production of sinterized details has been obtained. The granulometric distribution, technological characterization and microstructure analysis of the aluminium powder has been studied by means of the isothermic sintering of the pressing samples to different temperatures and retention times.
\end{abstract}

Keywords: Aluminium powder. Atomization. Powder metallurgy.

\section{INTRODUCCIÓN}

En la industria de la pulvimetalurgia, se utilizan 2.000 t/año de polvo de aluminio, lo que representa cerca del $1 \%$ del total de este polvo (1), que en su mayor parte se destina a la industria química por sus propiedades exotérmica y anticorrosiva, así como en la industria de la construcción en la fabricación de hormigón ligero. Sin embargo, en los últimos años se ha incrementado el interés por las piezas de aleaciones sinterizadas base aluminio, particularmente impulsado por la industria aeronáutica. Este interés se basa en que esta aleación es ligera y anticorrosiva, además de poseer propiedades mecánicas muy competitivas en relación con las aleaciones de base hierro.

(•) Trabajo recibido el día 16 de febrero de 1996

(*) Centro de Investigaciones Metalúrgicas (CIME). Avda. 51, 23611, Ciudad de La Habana (Cuba).

(**) Centro de Microscopía Electrónica, Universidad de Los Andes (Venezuela).

(***) Centro Nacional de Investigaciones Metalúrgicas (CSIC). Avda. de Gregorio del Amo, 8. 28040-Madrid (España).
Desde el punto de vista tecnológico, la condición principal del polvo de aluminio para su empleo en pulvimetalurgia consiste en tener una sinterabilidad alta, o sea, que la superficie de las partículas de polvo no debe formar una capa compacta de óxido de aluminio hidratado, lo que dificulta el proceso de difusión y sinterización entre las partículas.

Para cumplir esta condición, en el presente trabajo se describe la obtención de polvo de aluminio por atomización en la planta piloto del Centro de Investigaciones Metalúrgicas (CIME), de La Habana, utilizando nitrógeno como energía pulverizante y como agente para el enfriamiento. De esta manera, se espera obtener polvo de aluminio con un contenido de oxígeno inferior al $0,5 \%$ (2).

\section{PARTE EXPERIMENTAL}

\subsection{Obtención de polvo de aluminio}

El polvo de aluminio se obtiene en la planta de pulvimetalurgia del CIME cuyo esquema se muestra en la figura 1, que ha sido utilizada en otras investigaciones (3). 


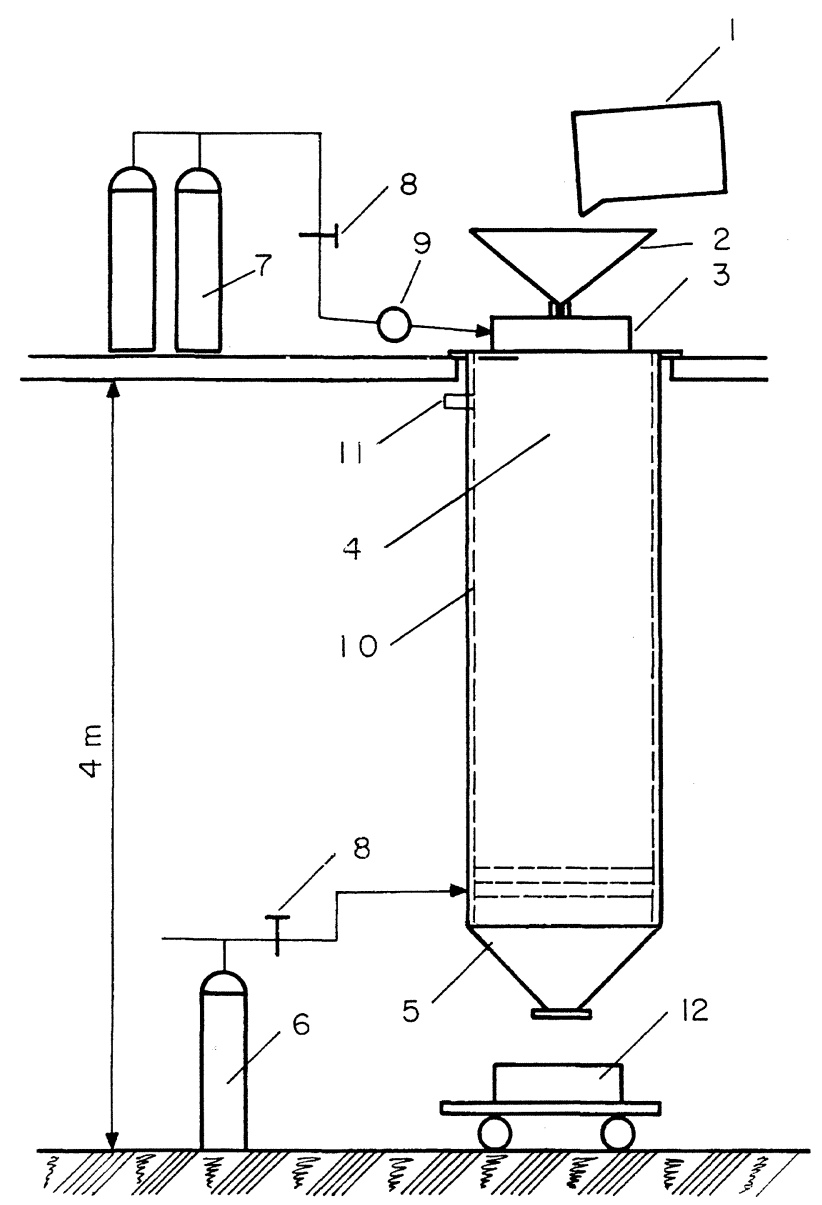

FIG. 1.- Instalación de pulverización: 1) Horno de inducción, 2) Metal líquido, 3) Inyector, 4) Cámara de pulverización, 5) Colector de polvo, 6) Gas $\mathrm{N}_{2}$ para enfriamiento, 7) Gas $\mathrm{N}_{2}$ para pulverización, 8) Regulador de presión, 9) Manómetro, 10) Camisa de agua, 11) Extractor y 12) Depósito de acero para recoger el polvo.

FIG. 1.- Powdering plant: 1) Induction furnace, 2) Liquid metal, 3) Injector, 4) Powdering chamber, 5) Powder collector, 6) Gas $N_{2}$ for cooling, 7) Gas $\mathrm{N}_{2}$ for powdering, 8) Pressure regulator, 9) Manometer, 10) Water jacket, 11) Extractor, and 12) Steel storage tank for powder

Como materia prima se emplea chatarra de aluminio con un $99,5 \% \mathrm{Al}$. Se prepara una carga material de $20 \mathrm{~kg}$, que se funde en un horno de inducción con crisol de grafito. Para desgasificar el baño de metal fundido, se le trata con una mezcla de fundente compuesta por $52,7 \% \mathrm{Ca}_{2} \mathrm{~F}$ y $47,3 \%$ $\mathrm{NaF}$, que previamente se homogeiniza durante 45 min y luego se envasa en cápsulas de aluminio. Cuando el metal líquido se encuentra a $800{ }^{\circ} \mathrm{C}$ se le adiciona el fundente en una relación de 0,2-0,5\% del peso de la carga, desgasificándose posteriormente con nitrógeno durante $5 \mathrm{~min}$. Inmediatamente, se atomiza con los siguientes parámetros:
- Temperatura del metal ….................. $800{ }^{\circ} \mathrm{C}$

- Diámetro del chorro de metal líquido $10 \mathrm{~mm}$

- Ángulo de incidencia ........................... $28^{\circ}$

- Presión del nitrógeno ........................ 0,5 $\mathrm{MPa}$

El chorro de metal se atomiza mediante un inyector con dos ranuras simétricas en forma de media luna y $1,3 \mathrm{~mm}$ de ancho cada una.

La atomización se produce dentro de la cámara de acero inoxidable con una camisa llena de agua para el enfriamiento de la cámara. La parte inferior de la cámara dispone de un serpentín para introducir $\mathrm{N}_{2}$ dentro de la cámara durante el proceso de atomización y enfriamiento.

El polvo, a una temperatura inferior a $50{ }^{\circ} \mathrm{C}$, se descarga desde el colector en una bandeja de acero inoxidable.

\subsection{Caracterización del polvo}

El polvo de aluminio se caracteriza mediante una serie de ensayos.

\subsubsection{Composición granulométrica}

Se determina según la Norma ISO 4497 "Metallic powders. Determination of particle size by dry sieving"; los resultados se ofrecen en la tabla I, en los que se observa que cerca del $80 \%$ de las partículas se encuentran en la fracción entre 210 y 1.600 $\mu \mathrm{m}$. El rendimiento de la fracción $<160 \mu \mathrm{m}$ es sólo del $16 \%$ y crece con el aumento de la presión de $\mathrm{N}_{2}$. El tamaño medio de las partículas, para una presión de $\mathrm{N}_{2}$ de $0,5 \mathrm{MPa}$, se calcula en $570 \mu \mathrm{m}$.

TABLA I.- Distribución granulométrica del polvo de aluminio, $\%$ en masa

TABLE I.- Granulometric distribution of the aluminium powder, \% in mass

\begin{tabular}{|c|r|}
\hline Fracción, $\mu \mathrm{m}$ & $\%$ \\
\hline $1600-\dot{8} 00$ & 31,10 \\
$800-630$ & 4,04 \\
$630-400$ & 9,54 \\
$400-315$ & 11,70 \\
$315-210$ & 21,68 \\
$210-160$ & 5,89 \\
$160-100$ & 11,45 \\
$100-80$ & 1,03 \\
$80-36$ & 1,56 \\
$>36$ & 2,01 \\
\hline \multicolumn{2}{|c|}{ Tamaño medio de las } \\
partículas de polvo: $570 \mu \mathrm{m}$ \\
\hline
\end{tabular}


Por cálculo se obtiene que el gasto específico de $\mathrm{N}_{2}$, para lograr este tamaño medio de partícula, es de $0,5 \mathrm{~m}^{3} / \mathrm{kg}$ de aluminio atomizado.

\subsubsection{Características tecnológicas}

Estas características se determinan según Normas ISO 2739 "Sintered metal bushes. Determination of radial crushing strength", ISO 3325 "Sintered metal materials, excluding hardmetals. Determination of transverse rupture strength", e ISO 4498/1 Sintered metal materials, excluding hardmetals. Determination of apparent hardness. Part 1. Materials of essentially uniform section hardness. El resultado se ofrece en la tabla II.

La densidad aparente del polvo es de 1,21 $\mathrm{g} / \mathrm{cm}^{3}$, valor semejante al de los polvos de aluminio empleados internacionalmente para uso en pulvimetalurgia (4 y 5).

La curva de compresibilidad del polvo se muestra en la figura 2. Con una presión específica de $4 \mathrm{t} / \mathrm{cm}^{2}$, se obtiene una densidad de $2,627 \mathrm{~g} / \mathrm{cm}^{3}$, equivalente al $97 \%$ de la densidad compacta. Con esta propiedad las muestras pueden ser sinterizadas en una gran variedad de atmósferas, y las propiedades de las muestras sinterizadas son buenas (6).

En comparación con los polvos de base cobre y hierro, el polvo de aluminio, debido a su morfología y a su baja densidad, tiene un índice de fluidez muy alto (fluye muy lento), del orden de $50 \mathrm{~g} / 100 \mathrm{~s}$.

\subsubsection{Difracción de rayos $X$}

El análisis de las fases del polvo de aluminio, con un mes de conservación en una atmósfera seca, se realiza mediante un difractómetro de rayos $\mathrm{X}$ DRON-3M. En el difractograma se observa la presencia de aluminio como fase única y, por tanto, no se detecta la presencia de $\mathrm{Al}_{2} \mathrm{O}_{3}$ en el polvo. Se puede considerar que el contenido de óxido de aluminio en el polvo es bajo, aunque de hecho este contenido puede ser grande en las partículas pequeñas donde la relación superficie/volumen es muy alta.

TABLA II.- Características tecnológicas del polvo de aluminio

TABLE II.- Technological characteristics of the aluminium powder

\begin{tabular}{|c|c|c|}
\hline $\begin{array}{l}\text { Densidad } \\
\text { aparente, } \mathrm{g} / \mathrm{cm}^{3}\end{array}$ & $\begin{array}{l}\text { Fluidez, } \\
\mathrm{s} / 50 \mathrm{~g}\end{array}$ & $\begin{array}{l}\text { Densidad a presión } \\
\text { de } 4 \mathrm{t} / \mathrm{cm}^{2}, \mathrm{~g} / \mathrm{cm}^{3}\end{array}$ \\
\hline 1,21 & 100 & 2,627 \\
\hline
\end{tabular}

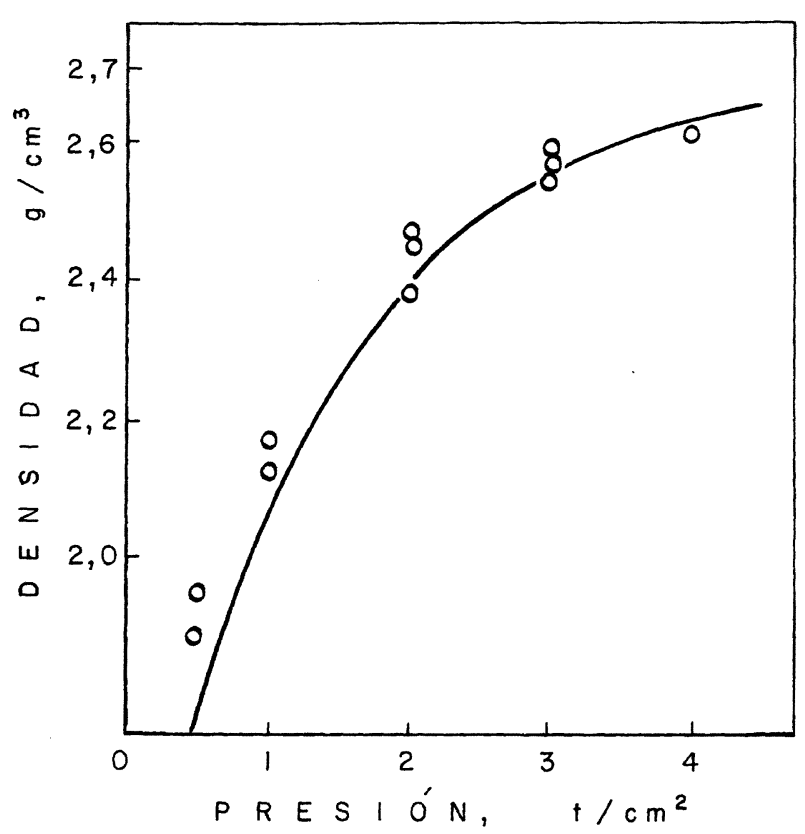

FIG. 2.- Curva de compresibilidad del polvo de aluminio.

FIG. 2.- Compressibility curve of the aluminium powder.

\subsubsection{Microscopía electrónica}

El estudio morfométrico de las partículas del polvo de aluminio se determina en un microscopio electrónico de barrido S-250.

En la figura 3 se puede observar que la forma de las partículas de polvo es muy diversa. La fracción fina, inferior a $100 \mu \mathrm{m}$, tiene forma redondeada y elipsoidal. En la fracción superior a $100 \mu \mathrm{m}$ hay abundancia de partículas con forma de filamento, con longitud de hasta $2 \mathrm{~mm}$ y $0,25 \mathrm{~mm}$ de anchura.

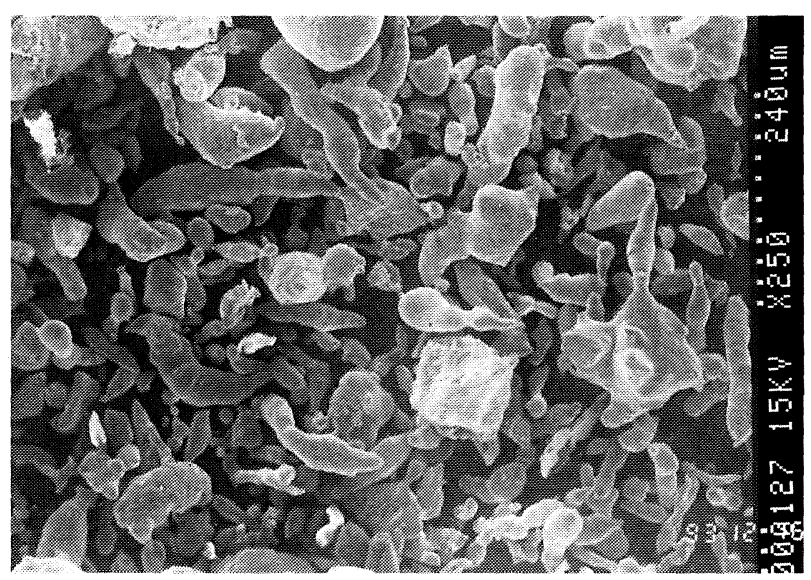

FIG. 3.- Microestructura del polvo de aluminio. $\times 240$.

FIG. 3.- Microstructure of the aluminium powder. $\times 240$. 
La figura 4 muestra con mayor detalle varias partículas localizadas en la figura anterior, con forma de filamento deformado o gota alargada.

El aspecto superficial que presenta la partícula de la figura 5, que se corresponde a la ampliación del filamento, es el típico de una partícula con estructura celular que inicia su solidificación en la superficie y donde las depresiones se deben al rechupe del líquido interdendrítico. Se conoce que las partículas de aluminio forman una lámina fina de óxido de aluminio durante la solidificación y enfriamiento de las partículas. El coeficiente de dilatación volumétrica del aluminio es mayor que el del óxido de aluminio (7), por lo que la contracción volumétrica de las partículas, durante el enfriamiento, forma una capa granulosa de óxido de aluminio.

Durante la observación de las partículas de aluminio, la atención principal se dirige a la búsqueda de poros que pudieran producirse a causa del atrapamiento de los gases durante la solidificación. En

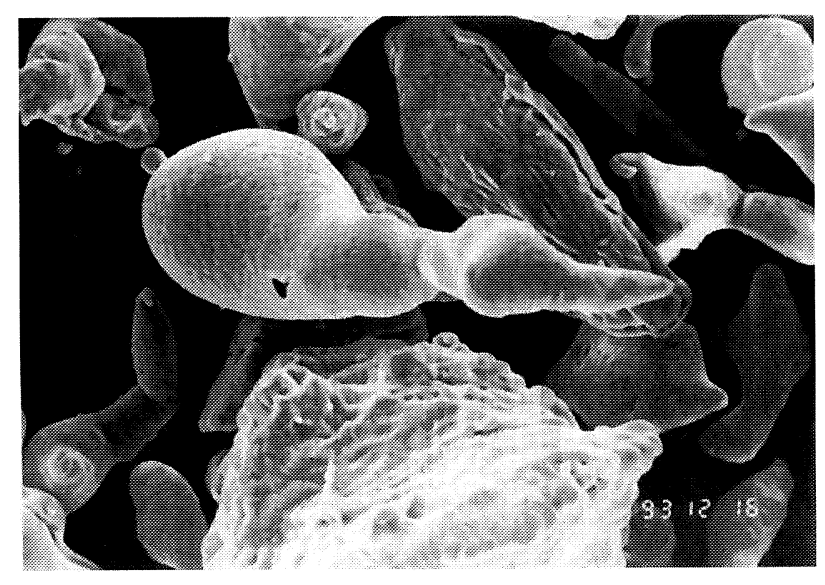

FIG. 4.- Detalle de una zona localizada en la figura 3. $\times 1.130$.

FIG. 4.- Detail of zone from figure 4. $\times 1.130$.

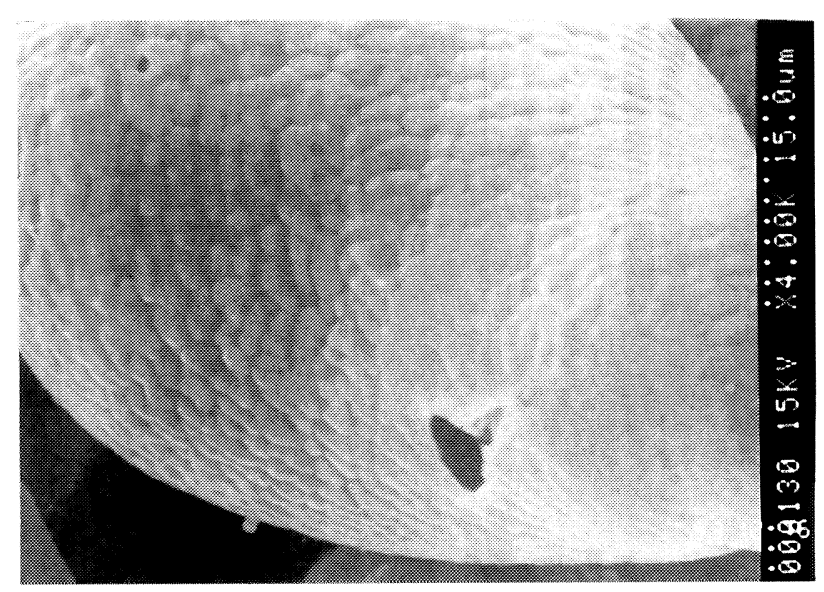

FIG. 5.- Detalle de una partícula localizada en la figura 4. $\times 4.000$.

FIG. 5.-Detail of particle from figure 4. $\times 4.000$. dependencia con el grado de contracción y localización de los poros, puede provocarse un efecto negativo en el proceso de sinterización, por ejemplo, la formación de ampollas y poros en la superficie de la pieza sinterizada. Normalmente, los poros se encuentran en las partículas gruesas, como se puede observar en las figuras 4 y 5 . Se puede establecer que la microestructura más representativa del polvo de aluminio consiste en partículas compactas, sin presencia de poros.

\section{SINTERIZACIÓN DEL POLVO DE ALUMINIO}

\subsection{Procedimiento}

El proceso de sinterización en estado sólido se manifiesta como el proceso de interacción de partículas sólidas, y se caracteriza fundamentalmente por el cambio de porosidad de la muestra con el consiguiente aumento de la compactabilidad.

El fenómeno más importante en la sinterización es la contracción de la muestra (8). Para estudiar este índice, en el presente trabajo se utilizan muestras en forma de briqueta que se someten a tratamiento en un horno tubular Griffin, con control automático de temperatura, con precisión de $\pm 0,5 \%$.

La contracción lineal de la muestra se mide con un micrómetro, después de la sinterización isotérmica, durante períodos de $1,3,5,8,12,20,30$ y 40 min en corriente de nitrógeno.

La elaboración de los datos experimentales se realiza mediante la siguiente ecuación (9):

$$
\frac{\Delta l}{l_{o}}=(K \cdot \tau)^{\mathrm{n}}
$$

donde:

$\Delta l=$ variación de la contracción lineal, $\mathrm{mm}$

$l_{\mathrm{o}}=$ longitud inicial, $\mathrm{mm}$

$\Delta l / l_{\mathrm{O}}=$ índice de contracción lineal

$\tau=$ tiempo de sinterización, $\min$

$K$ y $n=$ constantes que dependen de la temperatura, $\min ^{-1}$.

Las constantes $K$ y $n$ se determinan mediante el método de mínimos cuadrados, y aplicando un criterio establecido (10), se puede determinar el tipo de mecanismo de la sinterización. Este criterio se resume de la siguiente forma:

- Mecanismo de flujo viscoso .................. $r^{2} \sim \tau$

- Evaporación y condensación ................. $r^{3} \sim \tau$

- Depresión volumétrica ............................. $r^{5} \sim \tau$

- Difusión superficial ................................. $\quad r^{7} \sim \tau$ 
donde:

$r=$ radio del círculo de contacto entre dos partículas esféricas

El valor inverso de $r$ se corresponde con la constante $n$ de la ec. [1]. De la pendiente de la recta que se obtiene al representar $\ln \left(\Delta l / l_{\mathrm{o}}\right)$ frente a $1 / \mathrm{T}$, se calcula la energía de activación del proceso de sinterización $E$.

$$
K=K_{\mathrm{O}} \cdot \exp (-E / R T)
$$

siendo:

$E=$ la energía de activación, $\mathrm{J} / \mathrm{mol}$

$K=$ constante de la ec. [1], $\mathrm{min}^{-1}$

$K_{\mathrm{O}}=$ factor preexponencial, $\mathrm{min}^{-1}$

$T=$ temperatura absoluta de sinterización, $\mathrm{K}$

$R=$ constante de los gases ideales, $\mathrm{J} / \mathrm{K}$ mol

\subsection{Resultados}

En la tabla III se muestran las cinéticas de contracción, $\ln \left(\Delta l / l_{\mathrm{o}}\right)$ en función de $\ln \tau$, de las muestras sinterizadas en condiciones isotérmicas. De forma gráfica, esta dependencia se puede ver en la figura 6.

El valor medio calculado de la constante $n$ es 0,296 . El inverso de este valor es 3.38 , que se corresponde con el exponente de $\mathrm{r}$ para el mecanismo de difusión volumétrica y la evaporación-condensación, pero se acerca más al último.

El mecanismo de evaporación-condensación se caracteriza por la transferencia de átomos por la

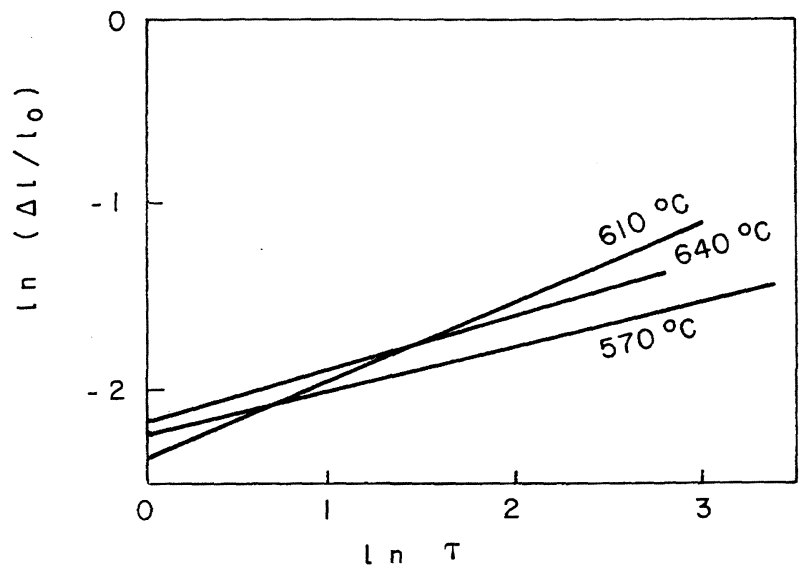

FIG. 6.- Representación de $\ln \left(\Delta l / l_{\mathrm{o}}\right)$ frente a $\ln \tau$ a diferentes temperaturas de sinterización.

FIG. 6.- Representation of $\ln \left(\Delta l / l_{o}\right)$ versus $\ln \tau$ to different sintering temperatures.

fase gaseosa producida con el cambio de presión de los gases, producto de la corriente de nitrógeno que se introduce en la zona de sinterización. De esta manera, el aumento del radio de contacto entre las partículas se produce básicamente por la evaporación de la superficie (libre, sin contacto) de la partícula y por condensación de este vapor en el punto de contacto entre las partículas.

De acuerdo con los resultados de la tabla III, se demuestra que durante el proceso de sinterización la muestra ensayada prácticamente no se contrae y el porcentaje de poros se mantiene.

TABLA III.- Dependencia entre el tiempo de sinterización $(\tau)$ y el índice de contracción $\left(\Delta l / l_{\mathrm{o}}\right)$ para el polvo de aluminio (desgasificado)

TABLE III.- Dependence between sintering time $(\tau)$ and contraction index $\left(\Delta \mathrm{l} / \mathrm{l}_{\mathrm{o}}\right)$ for the aluminium powder (degasificated)

\begin{tabular}{|c|r|l|l|l|c|}
\hline \multirow{2}{*}{$\begin{array}{c}\text { Temperatura de } \\
\text { sinterización, } \\
{ }^{\circ} \mathrm{C}\end{array}$} & \multicolumn{2}{|c|}{ Tiempo, min } & \multicolumn{2}{|c|}{ Contracción lineal } & \multirow{2}{*}{$n$} \\
\cline { 2 - 5 } & $\tau$ & \multicolumn{1}{|c|}{$\ln \tau$} & $\Delta l / l_{\mathrm{o}}, \%$ & $\ln \left(\Delta l / l_{\mathrm{o}}\right)$ & \\
\hline & 1 & 0 & 0,1212 & $-2,1186$ & \\
& 3 & 1,0986 & 0,1197 & $-2,1228$ & \\
& 5 & 1,6094 & 0,1190 & $-2,1286$ & 0,223 \\
& 8 & 2,0794 & 0,1779 & $-1,7265$ & \\
& 30 & 3,4012 & 0,2407 & $-1,4242$ & \\
\hline & 1 & 0 & 0,1220 & $-2,1037$ & \\
& 3 & 1,0986 & 0,1160 & $-2,1542$ & \multirow{2}{*}{640,396} \\
& 5 & 1,6094 & 0,1270 & $-2,0636$ & 0,396 \\
& 8 & 2,0794 & 0,2340 & $-1,4524$ & \\
\hline 640 & 15 & 2,7081 & 0,3490 & $-1,0527$ & \\
& 1 & 0 & 0,1203 & $-2,1178$ & 0,268 \\
& 15 & 2,7081 & 0,2480 & $-1,3943$ & \\
\hline
\end{tabular}


En la figura 7 se representa el valor de $\ln \left(\Delta l / l_{\mathrm{o}}\right)$ frente a $1 / T$. De la pendiente de la recta que se obtiene de esta representación se calcula el valor de la energía de activación: $E=105 \mathrm{~kJ} / \mathrm{mol}$.

\section{CONCLUSIONES}

- Se ha obtenido polvo de aluminio mediante la atomización del aluminio fundido con nitrógeno como energía pulverizante, y con posterior enfriamiento en corriente de nitrógeno. El polvo de aluminio presenta buenas propiedades tecnológicas y alta sinterabilidad.

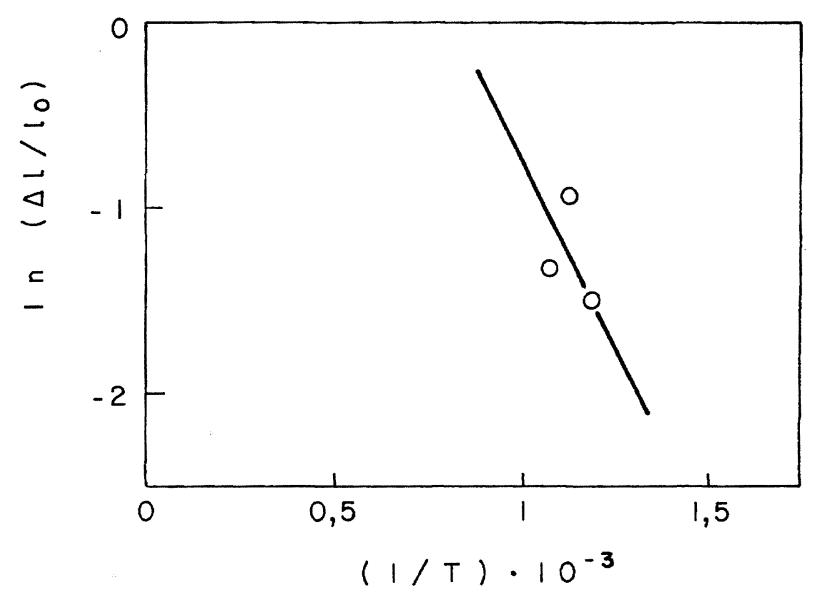

Fig. 7.- Representación de $\ln \left(\Delta / / l_{0}\right)$ frente a $(l / T)$ para la determinación de la energía de activación.

FIG. 7.- Representation of $\ln \left(\Delta l / l_{o}\right)$ versus $(l / T)$ for the determination of activation energy.
- La mayor presencia de partículas de polvo de aluminio en forma de filamento se produce debido a la baja presión de nitrógeno durante la atomización.

- El mecanismo de sinterización del polvo prensado se encuentra entre la difusión volumétrica y la evaporación-condensación.

\section{REFERENCIAS}

(1) Powder Metall. Int. Oct. 1993.

(2) LidDiARD, P.D. Powder Metall., 27 (4), 1984: 193-200.

(3) Krivij, N., Suwardjo, W. y Cores, A. Rev. Metal. Madrid, 31 (6), 1995: 394-399.

(4) Davies, B.L. y Farzin-Nia, F. Int. J. Powder Metall. Powder Technol., 19 (3), 1983: 197-209.

(5) Williams, J.E. Production of aluminium powder. Metals Handbook. Powder Metallurgy. Vol. 7. 5a Ed. 1984: 125.

(6) Storchneim, S. Precision Metall., 26 (6), 1968: 67, 68 у 73.

(7) Radomyselsky, I.D., Dovydenkov, V.A., DovydenkoVA, A.V. y KLimenko, A.I. Poroshk. Metall., (6), 1984: 82-90.

(8) Pines, B.Ya. J. Tech. Phys., 16 (6), 1946: 737-743.

(9) KisLi, P.S. y Kuzenkova M.A. Sinterización de los compuestos de alta temperatura de fusión. Ed. Naukova Dunka. Kiev, 1980 (en ruso).

(10) KucZynski, G.C. Trans. AIME, 1985 (2), 1949: 169-178. 